\title{
Information Sharing by Evacuee Collaboration for Assured Evacuation under Lack of Power Supply
}

\author{
Hisashi Ishii, Fumiko Harada and Hiromitsu Shimakawa \\ Graduate School of Science and Engineering, Ritsumeikan University \\ College of information Science and Enjineering, Ritsumeikan University \\ 1-1-1 Noji-Higashi, Kusatsu, 525-8577, Shiga, Japan \\ \{hisashi,harada,simakawa\}@de.is.ritsumei.ac.jp
}

\begin{abstract}
When a disaster occurs, it is very difficult for people inside a building to evacuate safely under lack of power supply. We propose a method to realize evacuation guide in case of power failure. In this system, passive RFID tags are put on walls of the building. Each user has a mobile terminal equipped with an RFID reader/writer. By referring to passive RFID tags which the user touched, the mobile identifies safe routes using the movement of people who have evacuated safely. The mobile terminal communication and RFID system realize collaboration of safety and dangerous routes among evacuees to share information of the routes.
\end{abstract}

\section{Introduction}

When disaster occur, it is very difficult for people inside a building to evacuate safely. If evacuees can realize collaboration of safety and dangerous routes, they can evacuate through safe routes and escape as possible as safely. In the cases of disasters, "collaboration" is regarded as sharing information on routes where there are fires and obstacles and information on routes which is safe. However, under lack of power supply, evacuees cannot realize collaboration to share their information because of server down and fire sensor troubles. For this reason, evacuees cannot recognize safety and dangerous routes, they may encounter dangerous ones. Under lack of power supply, sharing their information by collaboration among evacuees realizes a safe evacuation of people inside the building.

In this paper, we propose a system to realize collaborative evacuation to share information on safe and dangerous routes with an RFID system under lack of power supply. Our proposed method has the following three features:

- It uses passive RFID tags put on walls and mobile terminals equipped with RFID reader/writers.
- It can dynamically identify safe and dangerous routes from the information on routes where other evacuees passed through safely or went back.

- Even if direct communication is impossible in the building, it implicitly realizes collaboration to share their information which evacuees have.

We have partially implemented the functions of our proposed method to guide people to safe routes on the mobile terminals. We also have conducted an experiment to verify the efficiency of bringing mobile terminal by comparing increasing rate of blood pressure and heart rate before and after the evacuation, with and without mobile terminals. The experimental environment has a dead-end which evacuees do not know. Our purpose of setting this route is to cause states of confusion in the minds of the people who encounter the dead-end. In the experimental results, while average increasing rate of heart rate of evacuees who had encountered a dead-end without the mobile terminal was $21.9 \%$, that of evacuees with the terminal was only $4.0 \%$. This fact proves that the terminals bring assurance to evacuees.

The rest of this paper is organized as follows. Section 2 reveals problems of current evacuation guide systems and discusses the existing methods. Section 3 proposes the method to guide people to safe routes under lack of power supply. Section 4 shows an experimental result and its evaluation. Finally, Section 5 concludes this paper.

\section{Motivation}

\subsection{Anxious feeling for evacuees about dead or alive}

When a disaster occurs suddenly, it is very difficult for people inside a building to evacuate through safe routes. For this reason, it is possible that evacuees do not find escape 
exits in the building, also, they may fail to hear evacuation announces, they cannot recognize dangerous routes under lack of power supply or server down, they cannot communicate safe and dangerous routes which other evacuees found among evacuees. If evacuees encounter fires or obstacles, evacuees may lose their calmness for judgment. Therefore, evacuees need to be lead to safe routes under lack of power supply.

\subsection{Related works}

The purpose of our research is to guide people inside buildings to safe evacuation routes under lack of power supply.

Recently, several works has focused on appropriate identification of people location through many kind of sensors[1, 2]. Identification of locations has been applied to navigation systems. For example, a navigation system is proposed to guide people both inside and outside of homes by combining PDAs and car-navigation devices[3]. "Cochira" system developed by Japan Railway Corporation guides people to a destination with an RFID system[4]. Some researches have been proposed to guide with making consideration of crowded situations[5, 6]. However, these traditional navigation systems assume normal situations. Therefore, if a disaster occurs, these systems cannot realize to guide people to safe routes by making consideration of dangerous routes where there are fires or obstacles. Two existing systems guide people to avoid a critical situation when a disaster occurs[7, 8]. The first system[7] identifies dangerous routes from the movement speed of evacuees. In this system, RFID readers are equipped on the ceiling of a building and each evacuee brings an RFID tag. It identifies the speed of movement of a user by reading his RFID tags with some RFID readers. In the second method[8], situation of evacuees are identified through CCD cameras when disasters occur. The system can guide people to avoid crowded routes. However, since these systems use UHF RFID readers or CCD cameras which need power supply in the building, they cannot recognize situation of evacuees under lack of power supply. In the case that server down under lack of power supply, several servers which manage fire and smoke sensors cannot recognize dangerous routes and guide people to safe routes. Though there is an existing system that the mobile terminal equipped with permanent power supply guides each evacuee, this system needs power supply of infrared radiation sensors in the building[9]. Almost all of existing systems need power supply in the building. Therefore, these systems cannot always work well at everything when disasters occur. In addition, these systems cannot be realized with the low cost. It is extremely impossible that these systems are realized by balancing the cost of the building attendant with availability. Even if all terminals such as

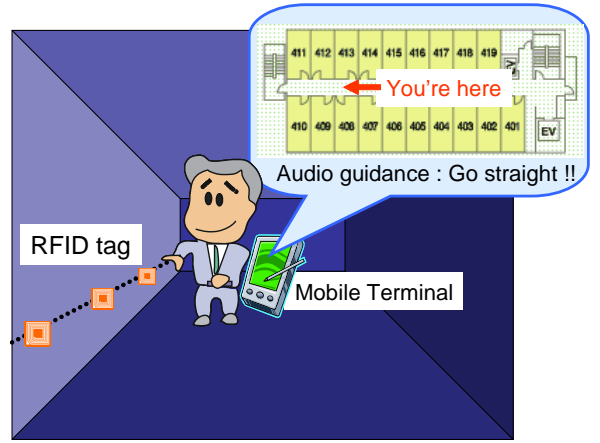

\section{Figure 1. Accommodation facility equipped with the RFID system}

severs, RFID readers and CCD cameras are equipped with batteries, we suggest that it is very difficult to realize an evacuation guide more costly than ever before.

\section{Evacuation guide in a power failure}

\subsection{Evacuation guide with an RFID system}

The proposed method assumes that the environment is the building where people have visited for the first time. We propose the evacuation guide with an RFID system as shown in Fig.1. We assume that each evacuee brings a mobile terminal equipped with RFID reader/writer. Many passive RFID tags embedded in sheets which store light energy are put on handrails and walls in the building. In case lack of power supply caused by a disaster, the sheets are luminous. Evacuees can be guided by holding the terminals over RFID tags in the darkness. Each tag has a unique ID of the location where the tag is put. Tags are read and written by the terminals. When an evacuee holds his mobile terminal over RFID tags, the terminal identify his current position from the ID written in the tag. The terminal selects an evacuation route and guides him to an exit with audio guidance and displaying a map.

\subsection{Graphing of building map}

To guide people from their current position to an exit in the building, the terminal must have detailed information on the construction of the building. We propose to represent the construction of the building as a route graph. Fig.2 shows how to represent path routes in a building as a route graph. Routes are described as a straight line graph. We link them with existing plot such as a room gateway or a stair. We define each intersection as a node. Each edge graph has a weight which represents current difficulty of passing 


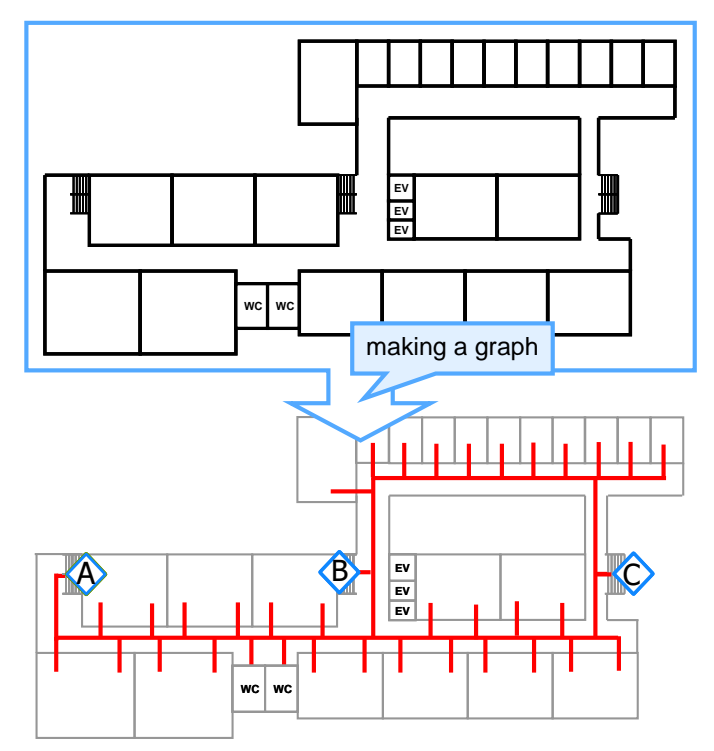

Figure 2. Making a graph from a floor map

through the route. The default weight in a route graph is set by a builder. The route graph of the floor inside the building is recorded in the terminal to search for the evacuation route from the current position to an exit. When an evacuee comes in a hotel, his mobile terminal receives the building map via wireless. It means that the mobile terminal stores the building map before he enters in the inner parts of the building.

Therefore, the terminal can guide a selected route which considered difficulty degree of passing through such as the differences in levels on the floors, the passageway width and so on.

\subsection{Searching for evacuation routes with tree graphs}

The mobile terminals are required to search for evacuation routes rapidly. Our proposed method considers that the terminals will be embedded in compact terminals such as mobile phones, watches and flashlights to alleviate a burden on having the terminals. To realize evacuation guide with compact ones which memory is limited strictly, this method is demanded to reduce computational load. Thus, we represent the route graph as a tree graph. The route graph is converted each edge of the route graph into a branch of a tree graph and a destination such as stairs and each exit into a root of each tree. The root of a destination is linked nearby the branch by turns. A cascade of the route graph does not include same branches not to use duplicative evacuation routes. We assume that all tree graphs for each destination are previously made. Tree graphs for destination

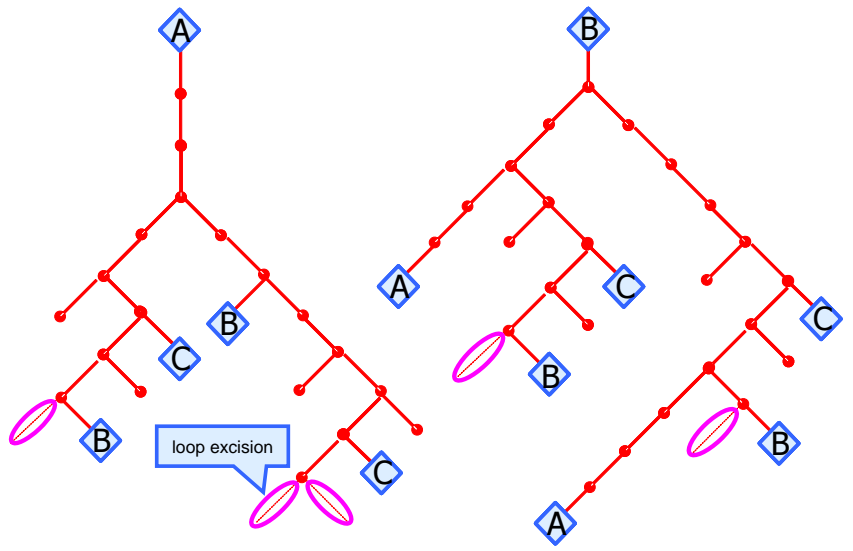

Figure 3. Tree structured graph

A and B in Fig.2 are represented as shown in Fig.3. Loop excision which is indicated in Fig. 3 prevent from guiding people the same evacuation routes. This system search for evacuation routes from current position of evacuees to only the destination. When an evacuee holds a terminal over an RFID tag, the terminal uniquely finds nodes of tree graph which indicate the current position of the tag. It selects an evacuation route by finding a path with the minimum total weight from the current node to the root.

Fig. 4 shows how to search for evacuation routes from the tree graph whose root is destination of $\mathrm{B}$. When the evacuee holds the terminal over an RFID tag, the terminal recognizes current position and defines current position as node $s$ on the tree graph in Fig.4. Current position s on the tree graph corresponds to s1 and s2. They are two evacuation routes which pass from $s$ to the root B in Fig.2. The total weight from s1 to destination $\mathrm{B}$ is calculated such as $10+10+10+5=35$. Similarly, the total weight from s2 is calculated as 95 . The system selects the evacuation route of s1 whose total weight is smaller than s2. By calculating only evacuation routes which track from the current position to the destination, this system can reduce the computational load. If we update the weight of each branch on tree graphs dependent on information of safe and dangerous routes, the terminal can dynamically change evacuation routes.

\subsection{Identifying safe routes based on collab- oration}

There is no way for the terminals to recognize dangerous routes because several sensors such as a fire sensor may be not able to detect dangerous routes under lack of power supply. In our system, the route is selected not to encounter dangerous routes by making consideration of evacuating through as safe route as possible and the distance from the current positions. Our system utilizes information of routes 


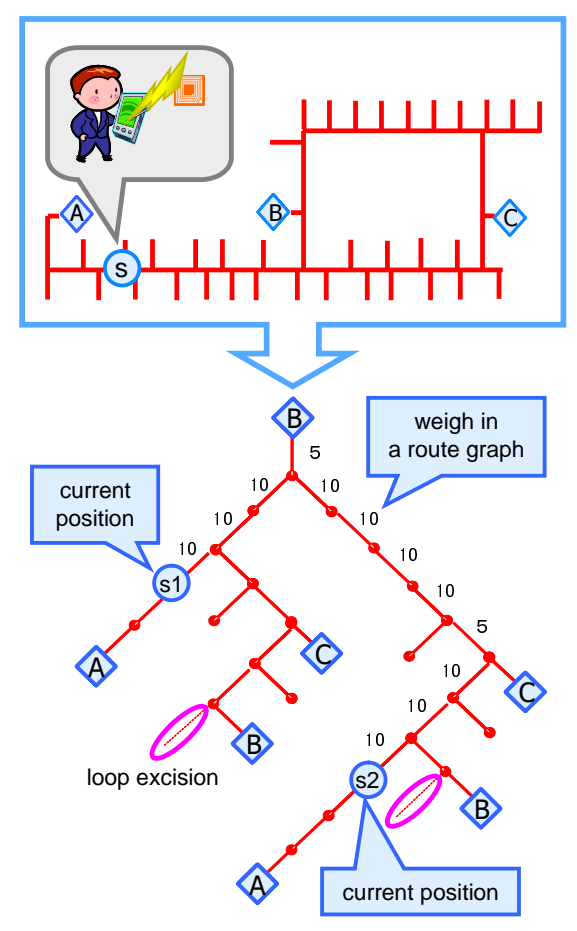

Figure 4. Searching for the evacuation route with tree graphs

which is obtained by movement of other evacuees. A route is regarded as a dangerous one if they went back in the route because of fires or obstacles. On the other hand, a route is regarded as a safe one if they passed through it and arrived at the destination such as stairs and exits. The terminal recognizes safe and dangerous routes from the history of the read tags for evacuation guide. Their information of routes is identified by how many other evacuees passed through or went back and when they passed through or went back. By reducing weights of tree branches on the safe routes and increasing weights of tree branches on the dangerous routes, the mobile terminal can guide people to safe routes.

\subsection{Collaboration among evacuees}

The mobile terminal cannot distinguish safe routes and dangerous routes completely by itself because it cannot utilize information of unknown routes that the user has never gone to under lack of power supply. Thus, we propose a collaboration to share the information of safe and dangerous routes among evacuees with mobile terminal communication and with writing into RFID tags. In the following, we describe how information are shared by mobile terminal communication and writing into RFID tags.

- Mobile terminal communication

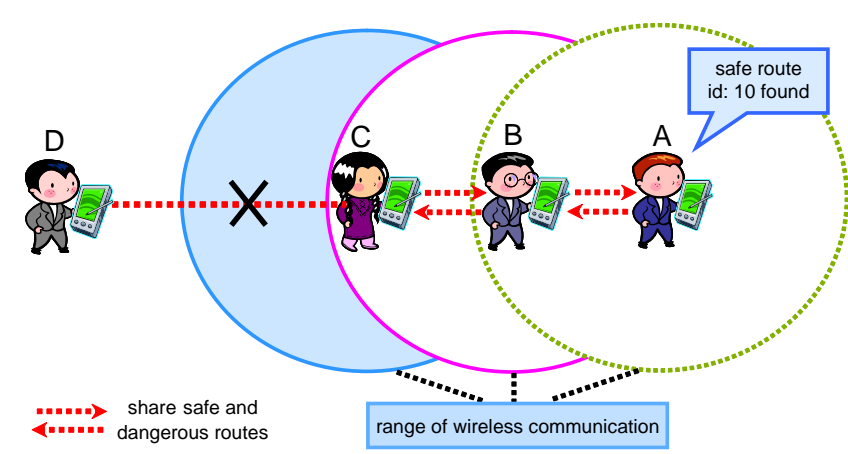

Figure 5. Mobile terminal communication

This method can share the information of safe and dangerous routes among mobiles in the wireless communication area of the mobile terminals. Fig. 5 shows an example of the mobile terminal communication such as Bluetooth that shares information of routes that evacuee A has gone. Evacuees B, C, and D are in the wireless communication area of the mobile terminals of A, B, and nobody, respectively. The A's terminal sends information of safe and dangerous routes to the B's terminal. This information includes the evacuee ID, routes' IDs, and timestamps when these routes are recognized as safe or dangerous ones. In Fig.5, evacuee A has the safe route of id 10. The B's mobile terminal updates information of safe route with id 10 according to the communicated information. Similarly, this information is shared between the mobile terminals of evacuees B and C. Therefore, a chained communication realizes sharing information of safe and dangerous routes with the other evacuees.

However, since evacuee D is in the wireless communication area of nobody, the D's mobile terminal cannot newly obtain information of routes from the others. To solve this problem, we propose writing into RFID tags.

- Writing into RFID tags

To support the mobile terminal communication, we propose writing the information of routes which each evacuee has obtained into RFID tags. Written information is about only dangerous routes because the capacity of RFID tags is limited and large capacity of written data many cause low speed of writing. Writing both of safe and dangerous routes may prevent an immediate evacuation. Fig. 6 shows an example of sharing information by writing into RFID tags. Though evacuee $\mathrm{C}$ cannot communicate with evacuee D in Fig. 5, writing into tags enables communication between evacuee $\mathrm{C}$ and $\mathrm{D}$ if they read from or write into an identical tag. 


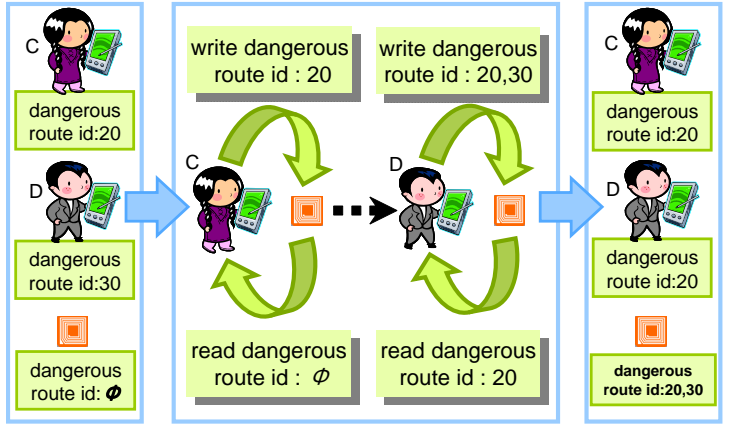

Figure 6. Writing into RFID tags

In Fig.6, the dangerous route with id 20 is stored in the C's mobile terminal. Also the dangerous route with id 30 is stored in the D's mobile terminal. The tag includes no route information at first. It is described by id $\phi$ in Fig.6. When evacuee $\mathrm{C}$ holds his mobile terminal over the tag, it recognizes current location and writes the dangerous route with id 20. After that, evacuee $\mathrm{D}$ holds his mobile terminal over the tag which evacuee $\mathrm{C}$ held it over. The D's mobile terminal reads the dangerous route with id 20 and writes the dangerous route with id 30. Many dangerous routes written by many evacuees may be stored in tags.

Therefore, mobile terminals can recognize dangerous routes without the mobile terminal communication and can guide users to safe routes.

\section{Experiment}

\subsection{Experiment objective}

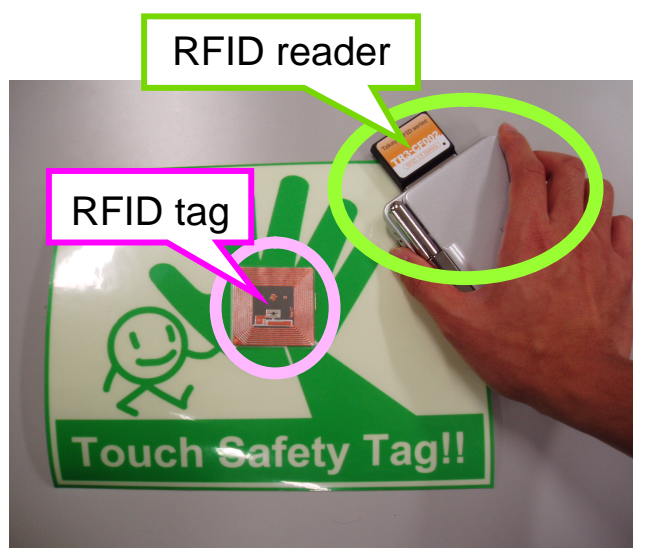

Figure 7. The mobile terminal and a RFID tag
We have to ensure that bringing the mobile terminal must be assured evacuees before we confirm the validation of our proposed collaboration. An evacuation system is required to give evacuees assurance since lives of evacuees are critical according to their mental condition when a disaster occurs. We should verify assurance of evacuees using the mobile terminals with the RFID system. We conducted an experiment to verify only availability of the mobile terminal without collaborating among evacuees. Fig.7 shows the RFID tag on the luminous sheet and the mobile terminal which we implemented to guide people to evacuate to safe routes. Though we should conduct experiment to verify availability of the mobile terminal in darkness, we did it in daytime because an experiment in darkness may make examinees involve a risk. Our experimental objective is to verify that evacuees with the mobile terminals are assured. If evacuees can evacuate safely, his mental condition is stable. If they encounter dangerous routes where a fire or obstacles exist, they cannot keep mental conditions stable. A mental condition affects on the blood pressure and heart rate. Therefore, in this experiment, we regard the blood pressure and heart rate as the mental condition of an evacuee. By comparing blood pressure, heart rate and time required for evacuation of people with the mobile terminals to that of evacuees without them, we verify this system.

\subsection{Experiment description}

Fig.8 shows the experimental environment. In the experiment, the starting point is in the 6th floor and the destination is in the 1st floor. This experiment conducts examinees as 9 evacuees who bring the mobile terminal and 7 evacuees who do not bring it. Evacuees who do not use the east stair do not usually know a dead-end of the east stair in Fig.8. To verify advantage of the mobile terminal, the deadend is previously set. RFID tags are put at about 4 to 8 meters intervals on the walls of the evacuation routes passing through the west and east stairs in Fig.8. The mobile terminal which we implemented can read tags at the communication range of 10 to 15 centimeters. Both evacuees with the mobile terminals and without them either evacuate the evacuation routes passing through east or west stair. We made the following rules explicitly for each evacuee:

- Evacuees with the mobile terminals

They walk holding the mobile terminals over RFID tags. They determine their evacuation routes according to the audio guidance of the mobile terminals. If they cannot hear the evacuation direction from the mobile terminals, they have to hold the terminals over the tag again. 


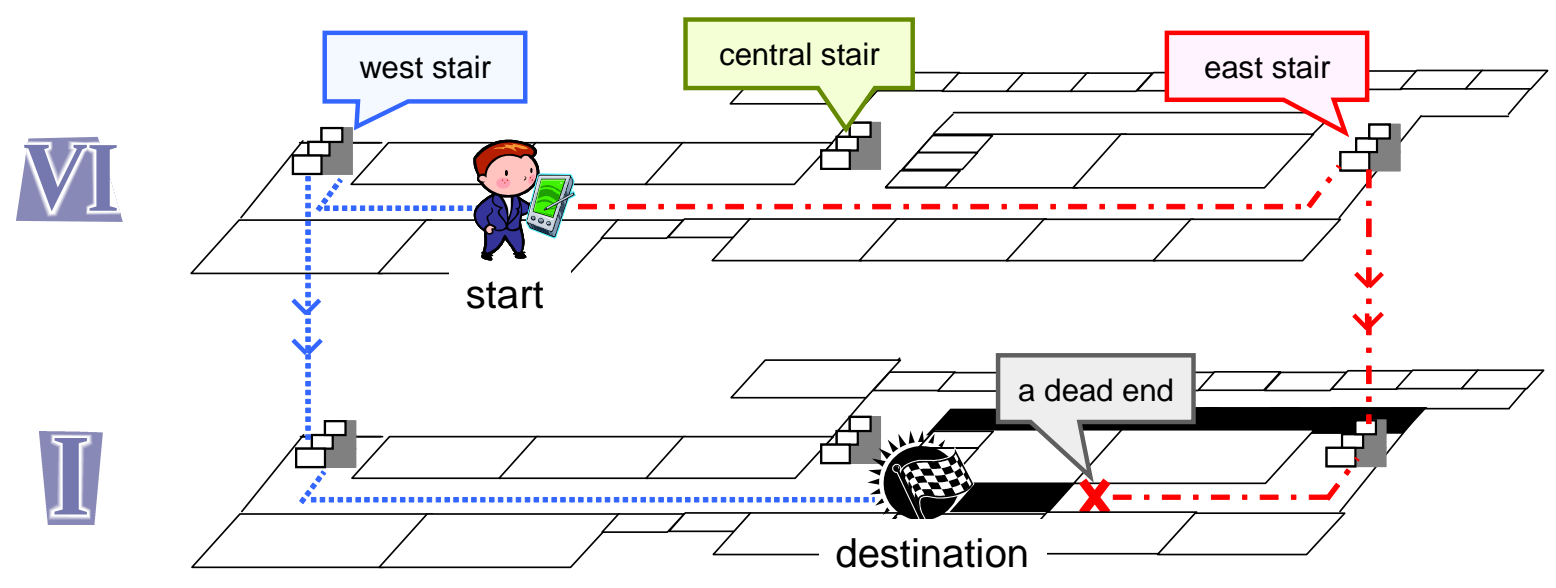

Figure 8. Experimental environment

- Evacuees without the mobile terminals

The supervisor previously instructed to evacuate through routes of which evacuees think as the shortest evacuation route from the starting point to the destination. In addition, he instructed that they do not intentionally evacuate to anomalistic routes and that they walk at their own pace.

At first, we measured blood pressures and heart rates of the evacuees at the starting point in Fig. 8 before they began evacuation. The supervisor measured the blood pressure and heart rate again and recorded the time required for evacuation when each evacuee arrived at the destination in Fig.8. Regardless of using the mobile terminals, all evacuees arrived at the destination without problems when they evacuate with passing through the west stair in Fig.8. On the other hand, evacuees without the mobile terminals encountered the dead-end in the 1st floor when they evacuated with passing through the east stair. Though they took it for granted that there are routes leading to the destination in the easterly direction, they encountered the dead-end and involved mental confusions. We consider that this situation corresponds to the mental condition of the evacuees who encounters dangerous routes where a fire occurs or obstacles exist in a disaster. When evacuees do not recognize a complicated structure of a building such as a hotel or an accommodation, they may be confused because they cannot find evacuation routes from current position to the exit by themselves. On the other hand, the evacuees with the mobile terminals are guided to the routes passing through the central stair just before they go down the stairs from the 2nd to the 1 st floor. Therefore, they can arrive at the destination without encountering this dead-end. We consider that evacuees with the mobile terminals are assured by them without changing rates of blood pressure and heart rate of evacuees heavily. We calculated increasing rates of blood pressure and heart rate of the evacuees with the mobile terminals and without them for each evacuation route. In our evaluation, we use average increasing rates of the blood pressures and the heart rates measured between before and after evacuation since we consider that the blood pressures and heart rates depend on an individual physical condition.

\subsection{Evaluation and review}

The experimental result is shown in Table 1. An increasing rate of the blood pressure and heart rate are the ratio of their values after the evacuation to their values before the evacuation. Median blood pressure is the average of maximum blood pressure and that of minimum blood pressure. By using this median blood pressure, we compared evacuees with the mobile terminals to evacuees without them. In Table 1, the average increasing rate of the median blood pressure of the evacuees without the mobile terminals and with them were $12.9 \%$ and $6.2 \%$ for the evacuees passing through the west stair, respectively. The average increasing rate of blood pressure of the evacuees without the mobile terminals and with them were $15.1 \%$ and $6.7 \%$ for passing through the east stair, respectively. Since the increasing blood pressure of evacuees with the mobile terminals was smaller than that of evacuees without the mobile terminals, using the mobile terminals could assure evacuees. In addition, increasing rate of heart rate of evacuees with the mobile terminals was also smaller than that of the evacuees without the mobile terminals. In Table 1, the average increasing rate of heart rates of the evacuees without the mobile terminals and with them were $7.2 \%$ and $6.0 \%$ for the evacuees passing through the west stair, respectively. Similarly, the average increasing rate of heart rate of the evacuees without the mobile terminals and with them 
Table 1. Experimental Result

\begin{tabular}{c|cc|cc}
\hline \hline \multirow{2}{*}{ increasing rate of } & \multicolumn{2}{|c|}{ without mobile terminals } & \multicolumn{2}{c}{ with mobile terminals } \\
\cline { 2 - 5 } & west route & east route & west route & east route \\
\hline maximum blood pressure & 1.118337 & 1.1167761 & 1.07357775 & 1.07362326 \\
$(+11.8 \%)$ & $(+16.8 \%)$ & $(+7.4 \%)$ & $(+7.4 \%)$ \\
\hline increasing rate of & 1.146019 & 1.126729 & 1.0471565 & 1.0600543 \\
minimum blood pressure & $(+14.6 \%)$ & $(+12.7 \%)$ & $(+4.7 \%)$ & $(+6.0 \%)$ \\
\hline increasing rate of & 1.128563 & 1.150537 & 1.0621289 & 1.06732 \\
median blood pressure & $(+12.9 \%)$ & $(+15.1 \%)$ & $(+6.2 \%)$ & $(+6.7 \%)$ \\
\hline increasing rate of & 1.072057 & 1.219274 & 1.059671 & 1.03997 \\
heart rate & $(+7.2 \%)$ & $(+21.9 \%)$ & $(+6.0 \%)$ & $(+4.0 \%)$ \\
\hline time required for evacuation $[\mathrm{sec}]$ & 137.915714 & 250.238571 & 224.547778 & 313.783333 \\
\hline
\end{tabular}

were $21.9 \%$ and $4.0 \%$ for the evacuees passing through the east stair, respectively. The increasing rates of blood pressures of the evacuees without the mobile terminals passing through the east stair shows a substantial rise while the increasing rate of blood pressures of them without the mobile terminals is very stable. However, this substantial rise of heart rates of the evacuees without the mobile terminals might be caused from the fact that evacuees lost their strength to go back since encountering the dead-end. However, in spite of distance of the east route which was longer than that of the west, the increasing rate of heart rate of evacuees who evacuated through the east route with the mobile terminals was smaller $2 \%$ than that of the evacuees who evacuated to the west route with them in Table 1. According to this review, it is high unlikely that distance heavily depends on the substantial rise $21.9 \%$ of increasing of heart rate of evacuees who evacuated through the east routes. We consider that one of the factors of the increment $21.9 \%$ is walking speed. Comparing the time required for evacuation with the mobile terminal to that of without them, the evacuees with the mobile terminals evacuated more slowly than without the mobile terminals. The deviation between increasing heart rates of the evacuees who evacuated through the west routes with the mobile terminals and without them was only $1.2 \%$. Therefore, we consider that another vital factor of the $21.9 \%$ exists. We obtained 6 evacuees in the 7 evacuees without the mobile terminals who encountered the dead-end from the questionnaire result of examinees. According to the questionnaire after the experiment, 6 evacuees were confused by wondering how to evacuate after they encountered the dead-end since the supervisor did not give any instructions. On the other hand, the heart rate of an evacuee who has known the dead-end route did not increase. All of the heart rates of other 6 evacuees increased and the average increasing rate of them was $25.6 \%$. This substantial increasing would cause by the case that the confused mental states caused by encountering the dead-end lasted until the evacuee arrived at the destination. In case of encountering the dead-end with real fires or obstacles, the heart rate of evacuees without the mobile terminals may increase more substantially than the experiment result. To avoid this situation, it is very important for evacuees to bring the mobile terminals. The experiment proves that the terminals bring assurance to evacuees.

In the experiment, the evacuees with the mobile terminals spent extra times to hear the audio guidance, by which evacuees might fail to escape. However, evacuees without the mobile terminals spend more extra time for evacuation to find safe evacuation routes under the darkness in the building such as a hotel or an accommodation of complicated structure. In the situation, our system has effectiveness by using the mobile terminal with audio guidance and luminous sheets in darkness.

\section{Conclusion}

We proposed an evacuation guide with an RFID system under lack of power supply when a disaster occurs. If evacuees can realize collaboration among evacuees, they can recognize information of unknown routes and can escape as safely as possible under lack of power supply. In this paper, we proposed a method that can guide people inside a building without power supply. Furthermore, our proposed method dynamically identifies safe routes by collaboration among evacuees. The RFID system and mobile terminal communication realize collaboration to share information of safe and dangerous routes under lack of power supply.

We have partially implemented the functions of our proposed method. We conducted an experiment to verify only availability of the mobile terminal. While the average increasing rate of heart rate of evacuees who encounter a dead-end without the terminals was $21.9 \%$, that of evacuees with the terminals was only $4.0 \%$. This fact proves that the terminals bring assurance to evacuees.

Future work aims to a complete implementation of our method. We will extend this collaboration system to share 
information of safe and dangerous routes among evacuees. Our future work will also aim to confirm the validity of collaboration among evacuees through a grand-scale experiment.

\section{References}

[1] J.J. Pan, J.T. Kwok, Q. Yang, and Y. Chen. Multidimensional Vector Regression for Accurate and Low-Cost Location Estimation in Pervasive Computing. IEEE Transactions on Knowledge and Data Engineering, Vol.18, No.9, pp.1181-1193, 2006.

[2] N. Davies, K. Cheverst, K. Mitchell, and A. Efrat. Using and Determing Location in a Context-Sensitive Tour Guide. IEEE Computer, Vol.34, No.8, pages 3541, 2001.

[3] A. Krüger, A. Butz, C. Müller, C. Stahl, R. Wasinger, K. Steinberg, and A. Dirschl. The Connected User Interface: Realizing a Personal Situated Navigation Service. Proceedings of the 9th International Conference on Intelligent User Interface, pages 161-168, 2004.

[4] T. Nakagawa, F. Tsunoda, K. Wakasugi, S. Isojima, and I. Saito. Development of Smart Navigation System " Cochira" for Customers in Railway Stations. Proceeding of the Ubicomp2005, 2005.

[5] H. Kawamura, K. Kurumatani, and A. Ohuchi. Modeling of Theme Park Problem with Multiagent for Mass User Support. Computer Science, pages 48-69, 2004.

[6] M. Ueda, R. Nishide, H. Hiura, M. Kawabata, and S. Ueshima. Proposing a Context-aware Pedestrian Navigation System and its Possible Applications. DBSJ Letters, Vol.4, No.1, pages 65-68, 2004.

[7] H. Ishii and H. Shimakawa. Evacuation Guide System with Visualization of Varying Dangerous Area and Person Movement. FIT2006, pages 3-4, 2006.

[8] H. Nakanishi, S. Koizumi, T. Ishida, and H. Ito. Virtual City Simulator for Transcendent Guidance. Proceedings of the 18th Annual Conference of the Japanese Society for Artificial Intelligence, pages 1-4, 2004.

[9] S. Oyama, T. Nishimura, I. Eguchi, and S. Igi. Design of the Audio Interface using CoBIT for Visually Impaired People. Proceedings of the 18th Annual Conference of the Japanese Society for Artificial Intelligence, pages 1-2, 2005. 